\title{
Polycomb response element-binding sites in the MDR of CLL: Potential tumor suppressor regulation
}

\author{
Christine E. Cutucache ${ }^{1}$, Javeed Iqbal'², Philip J. Bierman ${ }^{3}$, Robert Gregory Bociek ${ }^{3}$, \\ Dennis D. Weisenburger ${ }^{2}$, Shantaram S. Joshi ${ }^{4}$ \\ ${ }^{1}$ Department of Biology, University of Nebraska at Omaha, Omaha, USA \\ ${ }^{2}$ Department of Pathology and Microbiology, University of Nebraska Medical Center, Omaha, USA \\ ${ }^{3}$ Department of Internal Medicine Oncology/Hematology, University of Nebraska Medical Center, Omaha, USA \\ ${ }^{4}$ Department of Genetics, Cell Biology and Anatomy, University of Nebraska Medical Center, Omaha, USA \\ Email: ssjoshi@unmc.edu
}

Received 1 November 2012; revised 5 December 2012; accepted 10 January 2013

\section{ABSTRACT}

Chronic lymphocytic leukemia [CLL] is the most common adult leukemia and is heterogeneous in clinical presentation. CLL cases present with various chromosomal aberrations, including 11q23, 14q32, 17p, and trisomy 12, with the most common abnormality being deletion of 13q14 [1]. Although monoallelic deletion of 13q14 is common, there is a subset of patients who have complete nullisomy at 13q14, a locus that has been hypothesized to contribute to CLL pathogenesis [2] due to loss of tumor suppressors [DLEU and $m i R-15 a / 16-1]$. We hypothesized that deletion of both copies of 13q14 would lead to uncontrollable proliferation of CLL cells and a poor prognosis. We examined our 13q14 nullisomy for survival, treatment-free survival, lymphocyte doubling time, and the presence of lymphadenopathy. Furthermore, we compared the gene expression profiles between patients with 13q14 monosomy, nullisomy, or normal karyotype. Our results suggest that patients with $\mathbf{1 3 q}$ nullisomy have a higher incidence of bulky lymphadenopathy [16.6\% compared to $10 \%$ of monosomy patients], a higher frequency of lymphocyte doubling time $[27.7 \%$ compared to $7.4 \%$ of monosomy patients], and a higher rate of needing treatment [50\% compared to $18.5 \%$ of monosomy patients]. We observed deletion of DLEU1 and HTR2A, consistent with a gene dosage effect, and observed PRE-binding sites on DLEU1. Patients with homozygous deletion of 13q14 had a worse prognosis compared to heterozygotes. Lastly, the DLEU1 locus is a possible "second hit” loss for CLL progression.

Keywords: Chronic Lymphocytic Leukemia; Gene Expression; 13q14; Nullisomy; DLEU; Tolerogenic

\section{INTRODUCTION}

Chronic lymphocytic leukemia [CLL] is the most common adult leukemia and is heterogenous in clinical presentation. Previous studies have focused on identifying prognostic markers such as CD38- and ZAP70-expression, immunoglobulin mutational status, lymphocyte doubling time, presence of lymphadenopathy, and chromosomal abnormalities to better group patients for therapy $[1,2]$. Patients with CLL may have none, one, or several of the following chromosomal abnormalities: del [11q23], del [13q14], del [17p], trisomy 12, and, less frequently, del [14q32]. However, there exists a cohort of patients with loss of both alleles at $13 q 14$ [13q null] that is distinct of patients with monosomy at this region [13q mono] and wild type [WT]. The 13q14 locus was hypothesized to contribute to CLL pathogenesis due to the loss of tumor suppressors, DLEU and miR-15a/16-1 [3-6]. We hypothesized that deletion of both copies of $13 \mathrm{q} 14$ would lead to uncontrollable proliferation of CLL cells and a poor prognosis. To test this hypothesis, we performed retrospective analyses with the data we accumulated from CLL patients cared for at the University of $\mathrm{Ne}$ braska Medical Center [UNMC].

\section{PATIENTS AND METHODS}

\subsection{Patient Samples}

All peripheral blood CLL [PB-CLL] samples were obtained by venipuncture, and the CLL cells from bone marrow [BM-CLL] and lymph node [LN-CLL] were collected from the excess tissue samples. All protocols were approved by the Institutional Review Board [IRB] and Scientific Review Committee [SRC] at UNMC. PBCLL and BM-CLL cells were separated by using magnetic bead cell separation [Miltenyi Biotec, Auburn, CA, USA], and samples with $95 \%$ or greater CD5+CD19+ cells were used for analyses. To obtain LN-CLL cells, 
serial sections of frozen lymph node biopsies were obtained from the UNMC tissue bank. Immunohistochemistry was used to stain each section for CD5 and CD19 to identify areas of $>90 \%$ CLL cells. The CLL cells were microdissected out for the analyses.

\subsection{Fluorescent in Situ Hybridization [FISH] and Microarray Analyses}

Fluorescent in situ hybridization [FISH] was used to determine chromosomal abnormalities in CLL cells as previously described [7]. The annotated patient data was examined to determine the association between time to treatment, treatment-free survival, lymphocyte doubling time, and the presence of lymphadenopathy. Microarray analyses were used to determine differential expression of genes in a region of $13 q$ suggested to be deleted in CLL $[8,9]$.

Previously, a tolerogenic signature was associated with poor prognosis in CLL [9-12]. Genes from the tolerogenic signature were examined among patients with $13 \mathrm{q}$ null, 13q mono, and WT cases. Next, the genes within the 13q locus that were included in our array were clustered by CLL patients WT at this locus compared to $13 \mathrm{q}$ mono and 13q null. These results would describe whether there was a gene copy effect between CLL cases with losses at this region, which would identify a candidate tumor suppressor gene[s].

\subsection{Examination of Regulatory Elements}

The sequence for DLEU1 was examined using Vista [pipeline.lbl.gov/cgi-bin/gateway2] and the University of California, Santa Cruz [UCSC] Genome Bioinformatics [genome.ucsc.edu] Programs. The entire coding and noncoding sequence of DLEU1 was blasted for the polycomb response elements [PRE], including: GAF, G10, $\mathrm{PHO}$, and $\mathrm{Z}$ binding sites.

\section{RESULTS AND DISCUSSION}

\subsection{Prognosis for CLL Patients with 13q Nullisomy}

Our cohort of 13q null patients [ $\mathrm{n}=34$ ] had divergent clinical characteristics from that of $13 \mathrm{q}$ mono CLL cases $[\mathrm{n}=27]$. 13q null patients had higher incidences of bulky lymphadenopathy [ $16.6 \%$ null compared to $0 \%$ mono], shorter lymphocyte doubling time $[27.7 \%$ null compared to $7.4 \% \mathrm{mono}$, and greater occurrences of needing treatment (50\% null, $18.5 \%$ mono; Table 1). CLL patients were broadly defined as having a poor prognosis if there was lymph node-involvement, a short lymphocyte doubling time [defined as less than a year for doubling], and if they required treatment. Although CLL patients with $13 q$ deletion are generally classified as having a favorable outcome, we suggest a divergence in prognosis between patients with a heterozygous, compared to a homozygous, deletion at this locus. Accordingly, patients with homozygous deletion of 13q14 might benefit from earlier treatment.

We next examined the percentage of CLL cells with 13q null to determine their susceptibility to treatment. The FISH results from pre-treatment and post-treatment for CLL patients showed a decrease in the number of CLL cells positive for 13 q14 nullisomy from $18 \%$ pretreatment to only $5 \%$ patients after treatment $(\mathrm{p}=0.015$; Figure 1(a)). This $73 \%$ decline preliminarily suggests that early treatment of patients with $13 q$ null could be beneficial.

\subsection{Gene Expression Profiles [GEP] of Patients with 13q Nullisomy}

In order to determine whether there was a divergence in GEP between WT, 13q null, and 13q mono cases, microarray data were examined. Previously, we reported that a tolerogenic signature is indicative of patients with an unfavorable outcome compared to those with a more indolent disease [8]. Furthermore, based on clinical prognostic indicators, our results suggested that CLL patients with 13q14 null had a less favorable prognosis compared to those mono or WT. As a consequence, we examined the tolerogenic GEP between CLL cases WT, mono, or null at 13q14 to determine if this expression signature correlated with clinical outcome. We hypothesized that patients with $13 \mathrm{q} 14$ null would have higher expression of the tolerogenic signature compared to $13 \mathrm{q}$ mono or WT cases.

Changes in the GEP were compared between 13 null, 13q mono, and patients WT at the 13q14 locus (Figure 1(b)). There was an increase in the immunosuppressive

Table 1. Characteristics of CLL patients with 13q14 nullisomy compared to $13 \mathrm{q} 14$ heterozygous deletion.

\begin{tabular}{|c|c|c|c|c|c|c|}
\hline $\begin{array}{l}\text { Chromosomal } \\
\text { Aberration }\end{array}$ & $\begin{array}{l}\text { Avg Age at Diagnosis } \\
\text { Diagnosis (Range) }\end{array}$ & $\mathrm{N}$ (male) & $\%$ Patients with BLA & $\begin{array}{c}\% \text { Patients with }<1 \\
\text { yr LDT }\end{array}$ & $\begin{array}{c}\% \text { Patients ever } \\
\text { Treated }\end{array}$ & $\begin{array}{l}\text { Avg Months to } \\
\text { Treatment (Range) }\end{array}$ \\
\hline 13q14 wildtype & 63.15 years $(45-85)$ & $34(16)$ & $23.5 \%{ }^{*}$ & $17.6 \%$ & $56.3 \%$ & $40.75(1-245)$ \\
\hline 13q14 monosomy & 62.03 years $(46-79)$ & $27(13)$ & $0 \%$ & $7.4 \%$ & $18.5 \%$ & $57.1(1-185)$ \\
\hline $13 q 14$ nulilsomy & 63.1 years $(48-84)$ & $18(11)$ & $16.6 \%$ & $27.7 \%$ & $50 \%$ & $39.56(1-96)$ \\
\hline
\end{tabular}

*Indicates patients with favorable chromosomal abnormality, but with a high percentage of CLL cells expressing CD38; BLA: Bulky lymphadenopathy. 
(a)

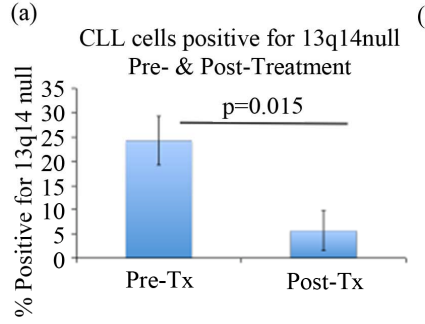

(b)

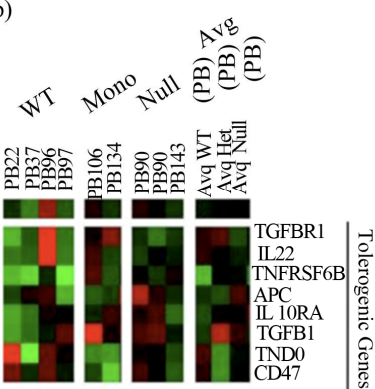

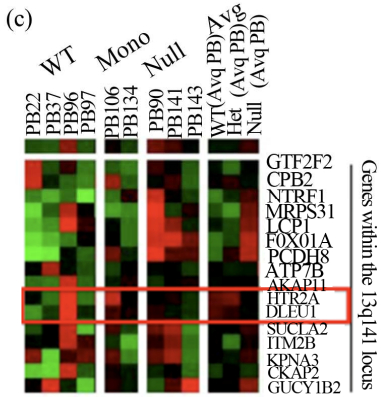

(d)

$13 \mathrm{q} 14$

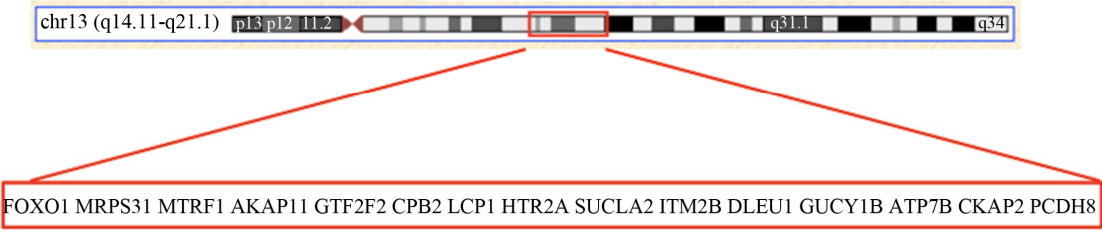

Deleted Region

Figure 1. Clinical and molecular characteristics of CLL patients with 13q14 nullisomy: (a) The change in the percentage of CLL cells positive for nullisomy at $13 \mathrm{q} 14$ pre- and post-treatment. The percentage of CLL cells positive for deletion at 13q14 as analyzed by FISH before and after therapy. $\mathrm{N}=5, \mathrm{P}=0.015$; (b) Tolerogenic gene expression profiles between CLL patients WT at the 13q14 locus, heterozygous (13q mono), or bearing a homozygous deletion (13q null). The genes in our microarray within this region were compared between patients who are WT, have a heterozygous deletion, or with a homozygous deletion at this region. Cluster analysis was performed using Cluster 3.0 and Treeview software; (c) GEP of genes at the 13q14 locus among CLL patients who are WT, have a heterozygous deletion, or with a homozygous deletion at this region. The genes in our microarray within this region were compared between patients who are WT, have a heterozygous deletion, or with a homozygous deletion at this region. Cluster analysis was performed using Cluster 3.0 and Treeview software; (d) Genes on 13q14 that were on our microarray chip (not an exhaustive list). Of the genes in the $13 \mathrm{q} 14$ region commonly deleted in CLL, the genes listed above were on our microarray. We were able to determine the gene expression profile for these select genes for CLL patients with both copies of $13 \mathrm{q} 14$ compared to those that were missing either one or both copies at this locus.

molecules $A P C$ and $T G F \beta 1$ in 13q null CLL cases when compared to $13 \mathrm{q}$ mono cases. $A P C$, a member of the Wnt signaling pathway, was shown to regulate cell migration and adhesion and colorectal cancer is associated with mutations in this gene $[13,14]$. TGF $\beta 1$ has been implicated in immunosuppression, in addition to cellular proliferation, differentiation, adhesion, and migration [1520].

\subsection{Gene Expression in CLL Cases Nullisomy, Monosomy, or WT at 13q14}

After determining a prognostic significance for complete loss of 13q14, the next objective was to identify whether a tolerogenic/immunosuppressive signature was differentially expressed in these cells. Supervised cluster analyses were performed on the genes included in our microarray platform that were within the 13q14 locus [8; MWG Biotech, Germany, Human 10K oligo set A; Fig- ure 1(c)]. The expected result was to observe a dosage effect of genes within the deleted region at 13q14. To determine critical genes in the region of interest, we expected patients WT at the locus to have high expression [red], those missing one copy [+/-] to have a lower expression [lighter red or green], and individuals null at this region to have no expression of critical genes [black]. We anticipated the results of these analyses would determine a region that was commonly deleted in CLL that we could compare to other reports in the literature.

The expression of two genes was consistent with the predicted expression pattern; these were $H T R 2 A$ and $D L$ $E U 1$ (Figure 1(c)). The location of HTR2A and DLEU1 is depicted in Figure 1(d). 5-hydroxytryptamine [serotonin] receptor 2A [HTR2A], G-protein-coupled receptor, has never been reported as having a role in CLL. Furthermore, as serotonin functions as a neurotransmitter, its association with cancer pathophysiology is an interesting observation. The gene deleted in lymphocytic 
leukemia 1 [DLEU1] is a long non-coding RNA [lnRNA] previously reported to participate in CLL [21-25]. Although there are only 15 published papers describing $D L E U 1$ to date, early reports and observations show promise that the regulation of this $\operatorname{lnRNA}$ appears to have a great influence on CLL progression.

Although the role of DLEU1 in CLL is still elusive, we searched the sequence of this region to gain greater insight. A polycomb response element [PRE] conserved sequence was identified, known to serve as an antagonist for epigenetic regulation of gene expression [26] within this region (Figure 2(a)). This region is a regulatory switchable element that influences the architecture of chromatin and the expression of nearby genes [27,28]. In this regard, $D L E U 1$ is potentially a docking site for the polyhomeotic [PHO] protein. This evolutionarily conserved, regulatory system was identified in Drosophila. The mammalian homolog of PHO in Drosophila is the

(a) DLEU1 (Partial sequence); Including PRE binding sites

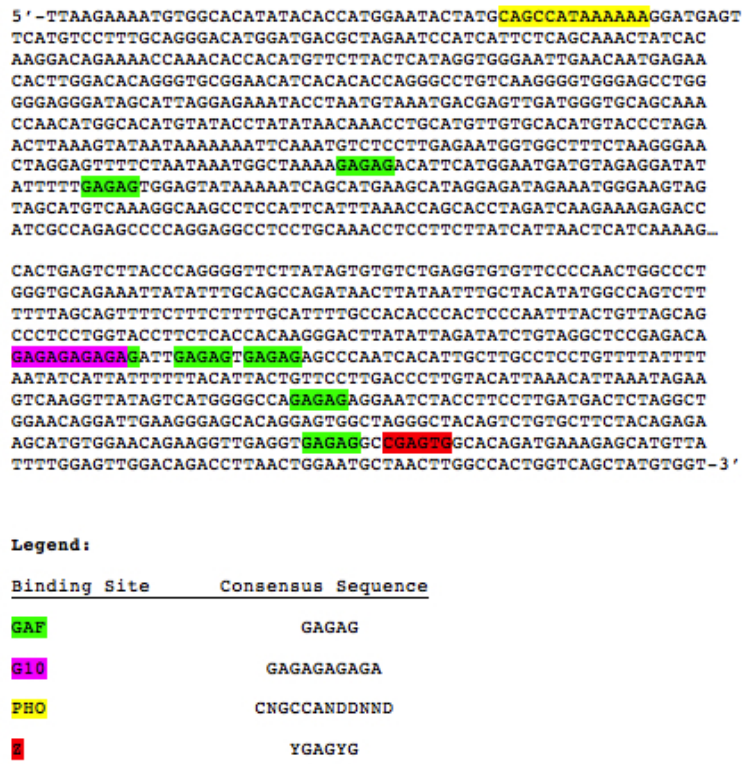

(b)
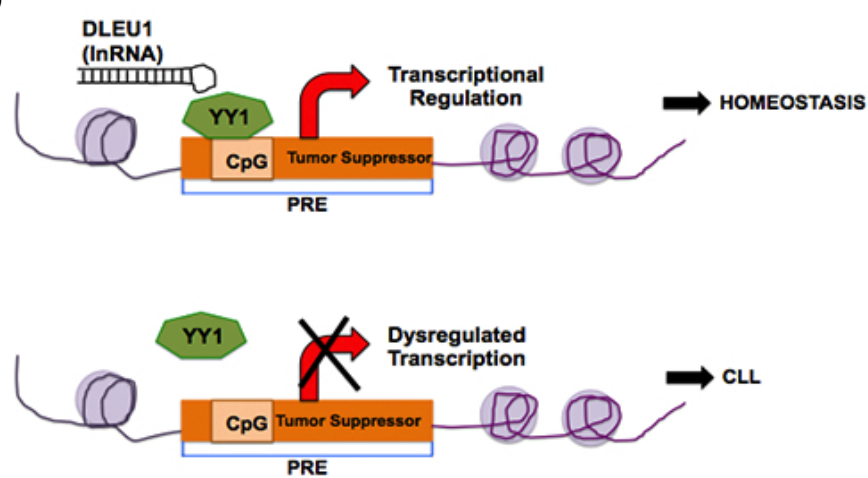

Figure 2. Polycomb response element binding site: (a) Putative PRE binding sites on the long noncoding RNA, DLEU1 at 13q14. The binding sites for the GAGA factor (GAF; 29), G10, extended GAGA site (G10; 30), PHO consensus (31), and Zeste binding site (Z; 30) are highlighted in the selected portion of the sequence as indicated by the legend. Due to the length of $D L E U 1$, an abbreviated portion of the sequence is provided in this figure; (b) Hypothesized mechanism of regulation by $D L E U 1$. DLEU1, a long non-coding RNA (lnRNA) deleted in CLL with unknown function. We have identified PRE binding sites on DLEU1 and describe a hypothesized mechanism of $D L E U 1$ regulating transcription to maintain homeostasis, but when $D L E U 1$ is lost as in the case of 13q null, then transcription is dysregulated and multiple "hits" to the 
genome likely contribute to CLL progression.

transcription factor $Y Y 1$ [29]. Recently, a lnRNA was the link between copy number variation and a polycomb/ trithorax epigenetic switch in muscular dystrophy [26], and we suspect a similar switch may be occurring in CLL following the loss of regulation of the region containing DLEU1 (Figure 2(b)). This finding suggests a potential negative feedback loop with the binding of $Y Y 1$ to $D L E U 1$ thereby regulating gene expression. Therefore, this finding would explain the over decade-long conundrum of observing a deletion in this region, but being unable to identify a clear point mutation or tumor suppressor gene.

In summary, this report describes an unfavorable prognosis for patients with biallelic deletion of 13q14, compared to $13 \mathrm{q}$ mono patients. However, the percentage of CLL cells with 13q null decreased after treatment, suggesting that chemotherapy was effective at killing these malignant cells. GEP of patients with $13 \mathrm{q}$ null, compared to $13 \mathrm{q}$ mono, identified the overexpression $A P C$ and TGF 1 . Therefore, the upregulation of these immunoregulatory molecules in $13 \mathrm{q}$ null patients might lead to a greater immunosuppression in these cases.

The limitation of this study is the number of cases examined. A larger cohort is needed to conclusively determine the biological relevance of DLEU1 in CLL cases with $13 q 14$ null, but hopefully this study will serve as a basis for additional in-depth analyses. Previously, a similar study with a large sample size $[\mathrm{n}=323]$ showed that a higher percentage of $13 \mathrm{q}$ null cells were associated with a significantly shorter time to treatment [6]. Similarly, we suggest that CLL patients with $13 \mathrm{q}$ null have a worse prognosis than patients that are mono or WT at 13q14. Contrastingly, these data contradict those described earlier this year [30]. Garg et al. observed that the baseline characteristics between CLL cases with mono- or bi-allelic deletion of $13 \mathrm{q}$ differed only by ZAP70-expression and albumin levels [31]. This study differs from ours in that solely fluorescent in situ hybridization [FISH] was used to assess the genetic abnormalities in CLL cells. Patients included in our study were first screened using both FISH and gene expression profiling. Therefore, this study presents a comprehensive picture as to the genetic abnormalities present within this frequently deleted region.

It will be difficult to prove a role for the consensus sequence of DLEU1 in CLL without engineering a deletion at that region both in CLL samples and an in vivo animal model. While this manuscript was in preparation, Lia et al. (2012) published a report regarding this precise question [31]. Transgenic mice engineered to have loss of $13 q 14$ had a shorter life expectancy, similar to our data from patients described in this report [31; Table 1]. The data presented herein suggest that, first, CLL cases show a heterozygous deletion at $13 \mathrm{q} 14$, and then a "second hit" or loss of the second allele [13q null] is necessary to take CLL from an indolent to an aggressive stage. Prospective studies are necessary to conclusively determine this switch causing CLL cells to drive a more aggressive disease.

\section{SUMMARY}

The findings presented herein are significant in that they suggest a different way of classifying CLL cases with $13 \mathrm{q}$ null. However, it is important to note the limitations with the sample size described in this study. Based on this small sample size, we suggest that patients with the deletion of both copies of $13 q 14$ potentially need therapy initiated earlier than their heterozygous counterparts and, thus, might require more frequent monitoring of lymphocyte counts. The influence of DLEU1 in CLL was reported previously, and the microRNAs in this region have been studied extensively [2-5]. However, this is the first report suggesting that regulatory elements, specifically PRE-sequences, of DLEU1 might contribute as a "second hit" to lead to a more aggressive disease progression for patients with CLL.

\section{ACKNOWLEDGEMENTS}

Thanks to the CLL patients who so willingly donated cells for our study. Also, thank you to the clinicians and nurses at the University of Nebraska Medical Center for collecting these samples.

\section{REFERENCES}

[1] Mraz, M., Pospisilova, S., Malinova, K., Slapak, I. and Mayer, J. (2009) MicroRNAs in chronic lymphocytic leukemia pathogenesis and disease subtypes. Leukemia \& Lymphoma, 50, 506-509.

doi:10.1080/10428190902763517

[2] Kasar, S., Salerno, E., Yuan, Y., et al. (2012) Systemic in vivo lentiviral delivery of miR-15a/16 reduces malignancy in the NZB de novo mouse model of chronic lymphocytic leukemia. Genes and Immunity, 13, 109-119.

doi:10.1038/gene.2011.58

[3] Lerner, M., Harada, M., Loven, J., et al. (2009) DLEU2, frequently deleted in malignancy, functions as a critical host gene of the cell cycle inhibitory microRNAs miR15a and miR-16-1. Experimental Cell Research, 315, 2941-2952. doi:10.1016/j.yexcr.2009.07.001

[4] Klein, U., Lia, M., Crespo, M., et al. (2010) The DLEU2/ miR-15a/16-1 cluster controls B cell proliferation and its deletion leads to chronic lymphocytic leukemia. Cancer Cell, 17, 28-40. doi:10.1016/i.ccr.2009.11.019

[5] Palamarchuk, A., Efanov, A., Nazaryan, N., et al. (2010) 13 q14 deletions in CLL involve cooperating tumor suppressors. Blood, 115, 3916-3922. doi:10.1182/blood-2009-10-249367 
[6] Van Dyke, D.L., Shanafelt, T.D., Call, T.G., Zent, C.S., Smoley, S.A., Rabe, K.G., Schwager, S.M., Sonbert, J.C., Slager, S.L. and Kay, N.E. (2010) A comprehensive evaluation of the prognostic significance of $13 q$ deletions in patients with B-chronic lymphocytic leukemia. British Journal of Haematology, 148, 544-550.

[7] Dickinson, J.D., Joshi, A.D., Iqbal, J., Sanger, W., Bierman, P.J. and Joshi, S.S. (2006) Genomic abnormalities in chronic lymphocytic leukemia influencing gene expression by a gene dosing effect. International Journal of Molecular Medicine, 17, 769-778.

[8] Joshi, A.D., Hegde, G.V., Dickinson, J.D., et al. (2007) ATM, CTLA4, MNDA, and HEM1 in high versus low CD38 expressing B-cell chronic lymphocytic leukemia. Clinical Cancer Research, 13, 5295-5304. doi:10.1158/1078-0432.CCR-07-0283

[9] Gilling, C.E., Mittal, A.K., Chaturvedi, N.K., et al. (2012) Lymph node-induced immune tolerance in chronic lymphocytic leukemia: A role for caveolin-1. British Journal of Haematology, 158, 216-231. doi:10.1111/j.1365-2141.2012.09148.x

[10] Mittal, A.K., Iqbal, J., Nordgren, T.M., et al. (2008) Molecular basis of proliferation/survival and migration of CLL in peripheral blood, bone marrow, and lymph nodes. Blood ASH Annual Meeting, 112, 546.

[11] Mittal, A.K., Gilling, C.E., Iqbal, J., et al. (2009) Clinical heterogeneity of CLL: Role for immune dysregulation mediated by the lymph node microenvironment. Blood ASH Annual Meeting, 114, 1243.

[12] Gilling, C.E., Mittal, A.K., Nganga, V., Palmer, V.L., Weisenburger, D.D., Bierman, P.J., Bociek, R.G., Swanson, P.C. and Joshi, S.S. (2010) Molecular determinants of lymph node microenvironment induced host immune tolerance in CLL: Role for CAV1, PTPN6, and PKC $\beta$ in the process. Blood ASH Annual Meeting, 116, 1367.

[13] Fang, Z., Xiong, Y., Li, J., Zhang, W., Zhang, C. and Wan, J. (2012) APC gene deletions in gastic adenocarcinomas in a Chinese population: A correlation with tumor progression. Clinical and Translational Oncology, 14, 6065. doi:10.1007/s12094-012-0762-X

[14] Valvezan, A.J., Zhang, F., Diehl, J.A. and Klein, P.S. (2012) Adenomatous polyposis coli (APC) regulates multiple signaling pathways by enhancing glycogen synthase kinase-3 (GSK-3) activity. The Journal of Biological Chemistry, 287, 3823-3832. doi:10.1074/jbc.M111.323337

[15] Bommireddy, R. and Doetschman, T. (2007) TGFbeta1 and $\mathrm{T}_{\text {reg }}$ cells: Alliance for tolerance. Trends in Molecular Medicine, 13, 492-501. doi:10.1016/j.molmed.2007.08.005

[16] Fogel-Petrovic, M., Long, J.A., Misso, N.L., Foster, P.S., Bhoola, K.D. and Thompson, P.J. (2007) Physiological concentrations of transforming growth factor beta1 selectively inhibit human dendritic cell function. International Immunopharmacology, 20, 1924-1933. doi:10.1016/j.intimp.2007.07.003

[17] Diaz-Valdes, N., Basagoiti, M., Dotor, J., et al. (2011) Induction of monocyte chemoattractant protein-1 and interleukin-10 by TGFbetal in melanoma enhances tumor infiltration and immunosuppression. Cancer Research, 71,

\section{2-821. doi:10.1158/0008-5472.CAN-10-2698}

[18] Bobr, A., Igyarto, B.Z., Haley, K.M., Li, M.O., Flavell, R.A. and Kaplan, D.H. (2012) Autocrine/paracrine TGF$\beta 1$ inhibits langerhans cell migration. Proceedings of the National Academy of Sciences of the USA, 109, 1049210497. doi:10.1073/pnas.1119178109

[19] Luo, H., Zhang, Y., Zhang, Z. and Jin, Y. (2012) The protection of MSCs from apoptosis in nerve regeneration by TGF $\beta 1$ through reducing inflammation and promoting VEGF-dependent angiogenesis. Biomaterials, 33, 42774287. doi:10.1016/j.biomaterials.2012.02.042

[20] Miron, R.J., Saulacic, N., Buser, D., Iizuka, T. and Sculean, A. (2012) Osteoblast proliferation and differentiation on a barrier membrane in combination with BMP2 and TGF $\beta 1$. Clinical Oral Investigations. doi:10.1007/s00784-012-0764-7

[21] Liu, Y., Corcoran, M., Rasool, O., et al. (1997) Cloning of two candidate tumor suppressor genes within a $10 \mathrm{~kb}$ region on chromosome $13 \mathrm{q} 14$, frequently deleted in chronic lymphocytic leukemia. Oncogene, 15, 2463-2473. doi:10.1038/sj.onc. 1201643

[22] Migliazza, A., Bosch, F., Komatsu, H., et al. (2001) Nucleotide sequence, transcription map, and mutation analysis of the 13q14 chromosomal region deleted in B-cell chronic lymphocytic leukemia. Blood, 97, 2098-2104. doi:10.1182/blood.V97.7.2098

[23] Wolf, S., Mertens, D., Schaffner, C., et al. (2001) B-cell neoplasia associated gene with multiple splicing (BCMS): The candidate B-CLL gene on $13 \mathrm{q} 14$ comprises more than $560 \mathrm{~kb}$ covering all critical regions. Human Molecular Genetics, 10, 1275-1285. doi:10.1093/hmg/10.12.1275

[24] Rondeau, G., Moreau, I., Bezieau, S., et al. (2001) Comprehensive analysis of a large genomic sequence at the putative B-cell chronic lymphocytic leukaemia (B-CLL) tumour suppresser gene locus. Mutation Research/Mutation Research Genomics, 458, 55-70. doi:10.1016/S0027-5107(01)00219-6

[25] Rowntree, C., Duke, V., Panayiotidis, P., et al. (2012) Deletion analysis of chromosome $13 \mathrm{q} 14.3$ and characterization of an alternative splice form of LEU1 in B cell chronic lymphocytic leukemia. Leukemia, 16, 1267-1275. doi:10.1038/sj.leu.2402551

[26] Cabianca, D.S., Casa, V., Bodega, B., et al. (2012) A long ncRNA linkes copy number variation to a polyco$\mathrm{mb}$ /trithorax epigenetic switch in FSHD muscular dystrophy. Cell, 149, 819-831. doi:10.1016/j.cell.2012.03.035

[27] Ringrose, L. and Paro, R. (2004) Epigenetic regulation of cellular memory by the polycomb and trithorax group proteins. Annual Review of Genetics, 38, 413-443. doi:10.1146/annurev.genet.38.072902.091907

[28] Schuettengruber, B., Chourrout, D., Vervoort, M., Leblanc, B. and Cavalli, G. (2007) Genome regulation by polycomb and trithorax proteins. Cell, 128, 735-745. doi:10.1016/j.cell.2007.02.009

[29] Simon, J.A. and Kingston, R.E. (2009) Mechanisms of polycomb gene silencing: Knowns and unknowns. Nature Reviews Molecular Cell Biology, 10, 697-708. 
[30] Garg, R., Wierda, W., Ferrajoli, A., Abruzzo, L., Pierce, S., Lerner, S., Keating, M. and O'Brien, S. (2012) The prognostic difference of monoallelic versus biallelic deletion of $13 \mathrm{q}$ in chronic lymphocytic leukemia. Cancer, 118, 3531-3537. doi:10.1002/cncr.26593
[31] Lia, M., Carette, A., Tang, H., et al. (2012) Functional dissection of the chromosome 13q14 tumor-suppressor locus using transgenic mouse lines. Blood, 119, 29812990. doi:10.1182/blood-2011-09-381814 